If a change to Mylan is considered necessary, take 1 hour before meals, not with meals, and monitor levels closely, especially with higher dose and blood levels.

Effect of food on bioavailability of Dilantin Kapseals. Dr Wilder and colleagues at the University of Florida have also examined the effect of food on a single $100 \mathrm{mg}$ dose of Dilantin Kapseals, given 1) after an 8-hour fast and 2) after a high-fat meal, in a nonblinded, randomized, crossover trial. (Cook J, Randinitis E, Wilder BJ. Neurology August (2 of 2) 2001;57:698-700). In 24 healthy adult volunteers, absorption of Dilantin was not significantly different under fasting and fed conditions. It is suggested that Dilantin Kapseals may be administered without regard to meal times, at least in healthy adults.

\title{
PAINFUL AURAS IN FOCAL EPILEPSY
}

The localizing and lateralizing value of painful epileptic auras in 25 patients with focal epilepsy were studied at the Cleveland Clinic, OH. In a group of 604 consecutive cases of focal epilepsies reviewed, 25 had painful auras (4.1\%). Age at seizure onset was mainly in childhood (median 7 years, range 1 to 42 ). Pain was associated with temporal (TLE, 14 cases), frontal (FLE, 6), parieto-occipital (POLE, 2), and perirolandic (PRE, 3) lobe epilepsies. The auras were localized to the abdomen (painful abdominal) in 10 (most with TLE), head and face (painful cephalic) in 11, and extremities (painful somatosensory) in 6. The greatest number of painful auras occurred with TLE, while the greatest frequency was with PRE (10\%). Although the number of painful abdominal auras was highest in TLE $(6 / 119,5 \%)$, the frequency was highest in FLE (4/8, 50\%). Somatosensory painful auras with PRE were contralateral to the epileptic focus, but with TLE they were sometimes ipsilateral. Painful epileptic auras may resolve after focal cortical resection, which suggests a cortical representation for pain. (Nair DR, Najm I, Bulacio J, Luders H. Painful auras in focal epilepsy. Neurology August (2 of 2) 2001;57:700-702). (Respond: Dr Dileep R Nair, Department of Neurology, Cleveland Clinic Foundation, 9500 Euclid Ave, Cleveland, $\mathrm{OH} 44195)$.

COMMENT. An ictal origin for pain has been described infrequently, originally by Gowers in 1901 and later by Lennox and Cobb in 1933. Among 750 patients reporting auras, $2.8 \%$ had painful sensations, vs $6 \%$ with numbness and $5.1 \%$ paresthesias (Lennox WG. Epilepsy and Related Disorders. Boston, Little, Brown, 1960). In the present series, the greatest number of cases were associated with temporal lobe epilepsy.

\section{GAIT-INDUCED EPILEPSY}

A 3 year-old boy with gait-induced seizures is reported from University of Navarra, Pamplona, Spain. His father and grandmother had primary generalized epilepsy. He sat late at 12 months and walked late at 26 months. After recovery from a febrile illness at 2 years 10 months, he developed jerking of the left lower limb, impairing is ability to walk. EEG showed right parietooccipital spikes, and generalized S/W in sleep. While walking, slow S/W rhythmic discharges at $\mathrm{C} 4$ correlated with jerking of the left leg. At rest, the discharges resolved. MRI was suggestive of polymicrogyria in right frontal area. Ictal SPECT showed hyperperfusion on the right side with walking. After valproate therapy for 3 weeks, jerking decreased and walking ability improved. (Iriate J, SanchezCarpintero R, Schlumberger E et al. Gait epilepsy. A case report of gait-induced seizures. Epilepsia August 2001;42:1087-1090). (Repints: Dr Jorge Iriate, Department of Neurology, Clinica Universitaria, Unioversity of Navarra, Pio XII 36, Pamplona 31008, Navarra, Spain). 